\title{
Disciplina, clima y desempeño escolar en Chile
}

\author{
Carolina Gazmuri, Jorge Manzi y Ricardo D. Paredes
}

RESUMEN

En este trabajo se evalúa el efecto de la influencia del profesor en el clima escolar dentro de la sala de clases mediante un análisis estadístico de observaciones de video en el aula, aplicado a 51.329 profesores. Se observa que ciertos aspectos del ambiente en la sala tienen una poderosa influencia en el rendimiento de los alumnos; en particular, que el manejo del grupo del curso que ejerce el profesor en el aula es más relevante y significativo que otras medidas del desarrollo de la clase. También se aprecia que el ambiente en el conjunto de las clases del establecimiento es un mejor predictor de los resultados de los alumnos que el ambiente en la sala de clases específica que reportan las pruebas de aprendizaje. Ello sugiere que mejorar el ambiente en la escuela es el desafío más relevante, aunque ello está menos al alcance de cada profesor individualmente.

PALABRAS CLAVE

CLASIFICACIÓN JEL

AUTORES
Educación, personal docente, rendmiento escolar, evaluación, Chile

A20, I21

Carolina Gazmuri es estudiante del Máster en Educación en el Teachers College de la Universidad de Columbia.cgazmurib@gmail.com

Jorge Manzi es profesor titular en la Escuela de Psicología de la Pontificia Universidad Católica de Chile. jmanzi@uc.cl

Ricardo D. Paredes es profesor titular en la Escuela de Ingeniería de la Pontificia Universidad Católica de Chile.rparedes@ing.puc.cl 


\section{I}

\section{Introducción}

En la mayor parte de los trabajos en que se analiza el rendimiento académico se consideran variables sociodemográficas de la escuela, pero escasamente el desempeño de los profesores. Ello se debe en parte a que las evaluaciones de estos son pocas e indirectas, y las observaciones en el aula prácticamente nulas. Más aún, se desconoce la existencia de análisis estadísticos sobre los efectos de la sala de clases, lo que obedece tanto al costo de hacerlo, como a la resistencia de los sindicatos de profesores y a lo incipiente que es la codificación de conductas en cuanto a permitir generar métricas sobre estas. No obstante, padres y expertos en educación en Chile identifican como el principal problema en la escuela a la "falta de interés de los alumnos" y "la falta de disciplina"; mientras que en encuestas se plantea que la disciplina es uno de los factores clave para los padres en sus decisiones de elección de escuela (Arancibia, 1994).

En este trabajo se identifican y cuantifican factores del clima en la sala de clases, diferenciando los determinados por el profesor de aquellos más asociables a la escuela, y que influyen en el rendimiento escolar. Para ello se dispone de la evaluación de más de 50.000 profesores de escuelas públicas en Chile, por medio de la observación a través de videos de una clase de cada profesor, los que han sido analizados y codificados por sicólogos y profesionales del área de educación.

Los autores agradecen los comentarios de un árbitro anónimo, el financiamiento del Proyecto CIE-01 de la Comisión Nacional de Investigación Científica y Tecnológica (CONICYT), y el financiamiento del Fondo Nacional de Desarrollo Científico y Tecnológico (FONDECYT), Proyecto 1140980.
Si bien en diversas investigaciones se han intentado detectar factores del ambiente en la clase que afectan al aprendizaje, ha prevalecido la observación puntual y un enfoque más sicológico, basados ambos en grupos pequeños de alumnos. El aporte de este trabajo es que se midió y cuantificó la influencia de algunos de los factores que se dan en el aula con una muestra de 51.329 observaciones, significativamente mayor que las reportadas en la literatura. Desde luego, esta visión complementa la más prevaleciente y de paso permite diferenciar el impacto de diversos factores que se dan en el aula, y que resulta difícil diferenciar desde la observación directa y el análisis de casos.

Cabe destacar que en este trabajo solo se incorpora un aspecto específico del clima escolar, que al menos teóricamente es manipulable por el profesor dentro de la sala de clases y que puede ser analizado mediante la observación de un módulo de clase. Además, solo se evalúa la relación de este aspecto del clima escolar con los resultados académicos de los alumnos medidos según pruebas estandarizadas. Se entiende, por cierto, que el clima escolar afecta a otros aspectos de la vida escolar de alumnos y profesores. Por último, cabe reconocer que el clima escolar es influido por la interacción de otros factores no observables que afectan a las observaciones de clima que se utilizarán en esta investigación.

El trabajo se estructura en tres secciones, aparte de esta Introducción. En la sección II se describe el contexto educacional en Chile en el que se produjo el avance hacia la evaluación de los profesores, y se revisa la literatura. En la sección III se plantea el modelo por estimar y en la sección IV se entregan las conclusiones. 


\section{II}

\section{Antecedentes}

\section{Contexto de la educación en Chile}

La educación ha sido uno de los principales temas para el Estado chileno y si bien ha habido una política de continuos cambios, hacia principios de los años ochenta se realizaron reformas que han marcado el desarrollo posterior y caracterizan la situación de la primera década del segundo milenio.

La reforma de comienzos del decenio de 1980 descentralizó la educación, traspasándose a las municipalidades los establecimientos educacionales estatales. A la vez, se cambió el sistema de financiamiento basado en los costos históricos, a un sistema de subsidio por alumno para que los estudiantes puedan asistir al establecimiento de su elección. Estas reformas se tradujeron en un aumento de la cobertura escolar, pero no lograron mejorar la calidad de la educación como era también uno de sus objetivos.

Si bien existen varios críticos al proceso de descentralización (Muñoz y Raczynski, 2007), Beyer (2009) argumenta que la descentralización no fue completa, ya que si bien los establecimientos no siguieron dependiendo del gobierno central, tampoco se dotó a los municipios de las capacidades necesarias para poder gestionarlos. En esta línea, el debate sobre la calidad relativa de la educación municipal ha sido intenso y la literatura abundante (véase Drago y Paredes, 2011).

La crisis económica que comenzó en 1981 condujo a una drástica reducción del presupuesto de la educación pública. Entre 1982 y 1990, el gasto público en educación cae en un 29\%. Hasta 1988 no existió en Chile ningún sistema de evaluación de la calidad educativa. Ese año se creó el Sistema de Medición de la Calidad de la Educación, más conocido como prueba SIMCE y que se aplica hasta el día de hoy. En su inicio, los resultados de esta prueba no eran de dominio público, pero en el año 1995 empiezan a ser publicados.

En el año 1990 se crea el Estatuto Docente, en el que se establecen remuneraciones mínimas para los profesores y el derecho a una carrera docente. Este genera un conjunto de rigideces para los establecimientos educacionales, particularmente debido a los límites a la movilidad y el despido de profesores. En 1991, se permite que las escuelas con financiamiento estatal lo complementen con cobros a los padres. Ello conduce a un elevado ingreso de escuelas privadas y reduce notoriamente la participación de la matrícula provista por escuelas municipales (Paredes y Pinto, 2009). Así, en el año 2008 había 11.905 establecimientos educacionales, de los cuales el $49 \%$ eran establecimientos municipales, el $44 \%$ establecimientos particulares subvencionados y un 6\% establecimientos privados (MINEDUC, 2008a).

La calidad de la educación en Chile se evalúa principalmente mediante la prueba SIMCE, cuyos resultados son comparables solo desde el año 1997 y a partir de ese año los puntajes fueron estables, sin mejoras significativas en los logros sino hasta 2010, cuando empiezan a mostrar mejoría. Las cifras dan cuenta de una gran inequidad en la calidad de la educación. Una parte relevante de alumnos después de 4,8 o 10 años de estudio no tienen los conocimientos y las habilidades básicas para el grado que cursan (Muñoz y Weinstein, 2009). En 2008, el 35\% de los estudiantes de $4^{\circ}$ básico fueron identificados como de nivel inicial en lectura y el $41 \%$ en matemáticas (obtenido de informes de los resultados nacionales, www.simce.cl).

Eyzaguirre y Le Foulon (2001) concluyen, a partir de los resultados de las pruebas de $4^{\circ}$ y $8^{\circ}$ básicos de 1999 y 2000 , que un porcentaje cercano al $40 \%$ de los alumnos de básica no son capaces de comprender lo que leen, y en segundo medio este porcentaje es del $33 \%$. En la prueba SIMCE de 1999 se obtuvo como resultado que el $32 \%$ de los alumnos de $4^{\circ}$ básico no han logrado las destrezas y conocimientos que debería tener un alumno de $2^{\circ}$ básico, el $25 \%$ tiene el nivel de un niño de $3^{\circ}$ básico, otro $25 \%$ domina inicialmente los conocimientos de $4^{\circ}$ básico y solo un $11 \%$ tiene los conocimientos adecuados.

Otras pruebas estandarizadas, como el Estudio Internacional de Tendencias en Matemáticas y Ciencias (TIMSS por sus siglas en inglés), muestran resultados similares. Uno de cada dos alumnos de $8^{\circ}$ básico tiene un retraso de por lo menos 4 años en matemáticas. Además, los alumnos chilenos provenientes de hogares de altos recursos educativos, y que por lo tanto registran un mejor desempeño que el resto de los estudiantes del país, obtienen un promedio inferior al promedio general en esta prueba y su desempeño es del mismo nivel que el de alumnos de bajos recursos de la República de Corea, Eslovenia, la Federación de Rusia, Bélgica y otros países. Pero no solo la calidad promedio es baja, 
sino que también hay un problema de desigualdad. En efecto, entre los alumnos que asisten a escuelas privadas, 1 de cada 2 obtiene más de 300 puntos en la prueba SIMCE de matemáticas; en cambio, de los alumnos de escuelas particulares subvencionadas 1 de cada 5 logra ese rendimiento y, por último, de los alumnos de escuelas municipales solo 1 de cada 10 logra los 300 puntos (Fontaine, 2002; Brunner y Cox, 1995; García y Paredes, 2010).

\section{Situación de los docentes}

El planteamiento de Barber y Mourshed (2008), en el sentido de que "la calidad de un sistema educativo tiene como techo la calidad de sus docentes" (pág. 15), ha inspirado a distintos gobiernos para centrarse en la situación docente, empezando por hacer más atractiva la carrera. En el caso de Chile, ello es evidente. La mayor parte de los profesores del país no fueron los mejores alumnos en su etapa escolar, y de aquellos profesores más jóvenes solo 1 de cada 24 que ingresaron a la carrera de Pedagogía en las universidades del Consejo de Rectores de las Universidades Chilenas pertenece al $10 \%$ superior de los alumnos de su generación en la escuela (Claro, 2009). Ello se corrobora en Cabezas y otros (2013), quienes advierten sobre la necesidad de mejorar la dotación docente, sobre todo en el caso de las escuelas donde se observa mayor vulnerabilidad.

En el año 2008, en Chile ejercían 176.472 docentes, de los cuales el $46 \%$ pertenecían al sector municipal, el $43 \%$ al sector particular subvencionado y solo un $11 \%$ al sector particular. La mayoría de los docentes son mujeres $(71 \%)$, y las rentas del magisterio han aumentado alrededor de un $200 \%$ entre 1990 y 2008, pero este incremento no ha dependido del desempeño individual (MINEDUC, 2008a).

La idea de que la calidad de la docencia era la "piedra de tope" del aprendizaje de los niños motivó que, a partir de 2003 y luego de una larga negociación con el Colegio de Profesores, se iniciara una Evaluación Docente individual para los profesores de los establecimientos del sector municipal. El diseño de la Evaluación Docente tuvo un alto componente político y de negociación, por lo que podría esperarse que no evaluara bien el desempeño de los profesores. Sin embargo, al contrario de lo esperado, León, Manzi y Paredes (2008) encontraron una alta correlación condicionada de los resultados de las evaluaciones de los profesores con los aprendizajes que tienen sus alumnos. Esto sugiere que el diseño de la evaluación ha sido positivo.

\section{Clima y disciplina escolar}

El clima, el manejo de la sala y la disciplina son aspectos que suelen considerarse cruciales en el aprendizaje (véanse, por ejemplo, Ritter y Hancock, 2007; Nie y Lau, 2009 y referencias). Aunque esos conceptos han sido definidos de distintas formas en la literatura, en general, en todas las definiciones se consideran aquellas acciones adoptadas por el profesor para establecer el orden, involucrar a los estudiantes u obtener su cooperación dentro de la clase (Emmer y Stough, 2001).

Kennedy (2005) sugiere que frecuentemente el manejo del grupo en la clase interfiere con la capacidad del profesor para manejar las ideas que quiere transmitir a los alumnos. Explica que los profesores, temiendo perder la atención de los alumnos, sacrifican el compromiso intelectual de estos para mantener el orden con más facilidad. Ello, porque presentar los contenidos con mayor profundidad suele implicar un compromiso intelectual más elevado, y puede provocar que algunos alumnos pierdan el interés o se distraigan debido al mayor esfuerzo que deben hacer para seguir la clase, lo que puede generar desorden en la sala.

También el clima y la disciplina en la clase se han identificado como un factor crítico en la satisfacción laboral de los docentes. Constantemente, los profesores mencionan la disciplina escolar como uno de los desafíos más duros que tienen que enfrentar (Ritter y Hancock, 2007). En esta línea, los problemas de disciplina en los alumnos suelen considerarse como una de las principales razones que motivan a los docentes a abandonar la profesión (Morris-Rothschild y Brassard, 2006).

En atención a la importancia que tiene el clima para el resultado académico de los alumnos y para la satisfacción laboral de los profesores, en diversos estudios se ha tratado de identificar estilos de disciplina y evaluar su efectividad. En la literatura se identifican principalmente tres estilos de disciplina (Lewis y otros, 2008). En el primero se sugiere que el profesor debe tener un alto control de la sala de clases y del comportamiento de sus alumnos. En esta línea se incluye la "disciplina asertiva", concepto desarrollado por Lee y Marlene Canter a partir de 1970 (Malmgren, Trezek y Paul, 2005). En este estilo disciplinario se plantea que los profesores, al comienzo del año escolar, deben establecer las expectativas de comportamiento de los alumnos y las consecuencias de no cumplirlas. Además, durante las clases los profesores deben aplicar premios y reconocimientos por los buenos comportamientos y castigos para aquellos alumnos que incurran en faltas de disciplina. 
En esta misma línea, el "estilo intervencionista" sugiere que los estudiantes aprenden a comportarse en la sala de clases cuando su buena conducta es reforzada con premios y el mal comportamiento con castigos; sugiere por lo tanto que los docentes tengan un estricto control sobre las actividades de los estudiantes en el aula (Ritter y Hancock, 2007).

Una segunda línea pone mayor énfasis en el autodominio de los alumnos y da menor importancia a la autoridad del profesor. A este tipo de disciplina pertenece el Teacher Effectiveness Training, modelo desarrollado por Thomas Gordon, también durante los años setenta. Este estilo se basa en el autodominio de los propios alumnos para lograr el buen comportamiento del curso, y este autodominio se debe lograr negociando y conversando con ellos (Malmgren, Trezek y Paul, 2005). En esta dirección apunta el estilo "no intervencionista", que sugiere que a los estudiantes se les debe permitir ejercer una importante influencia en la sala de clases y que los profesores no deberían preocuparse por ajustar el comportamiento de los alumnos a lo que ellos consideran adecuado, ya que estos tienen un impulso interno que tenderá al comportamiento adecuado (Ritter y Hancock, 2007).

Por último, la tercera línea pone el énfasis en la participación y la toma de decisión del grupo y son los alumnos quienes deben tomar la responsabilidad del comportamiento de sus compañeros en la sala y asegurar que este sea correcto. Esta línea se basa en lo desarrollado por William Glasser en su teoría de control (Control Theory). Para el desarrollo de este estilo disciplinario, es importante la realización de reuniones de curso donde se discuten distintos temas de comportamiento y se buscan consensos al respecto (Edwards y Mullis, 2003).

En los países menos desarrollados, y en Chile en particular, hay pocos estudios sistemáticos, aun cuando en el de Eyzaguirre y Fontaine (2008) se sugiere que los profesores en las escuelas de alto rendimiento escolar dedican más tiempo de la clase a trabajar en materias instruccionales, lo que logran con una mayor planificación de las actividades, un mejor manejo de la disciplina y políticas de nivelación.

\section{Evaluación Docente en Chile}

La Evaluación Docente que se realiza a los profesores de las escuelas municipales de Chile quedó establecida en el Estatuto Docente promulgado en 1991. Sin embargo, debido a la gran oposición por parte del Colegio de Profesores, esta no pudo ser implementada de manera individual sino hasta 12 años después, cuando en 2003 el gobierno logró un acuerdo con dicha entidad. El acuerdo consiste en una evaluación, llamada Sistema de Evaluación del Desempeño Profesional Docente, que se les realiza a los profesores del área municipal del país. Esta evaluación incluye cuatro instrumentos con las siguientes ponderaciones: i) Autoevaluación (10\%); ii) Entrevista por un evaluador par (20\%); iii) Reseña del director o del jefe de la Unidad Técnico Pedagógica (10\%), y iv) Portafolio (60\%).

El Portafolio es un instrumento de evaluación en que el docente debe presentar evidencia de su práctica pedagógica y está compuesto de dos módulos. El primer módulo consiste en la descripción de una unidad pedagógica, una evaluación de dicha unidad y una reflexión pedagógica. El segundo módulo contempla la filmación de 40 minutos de una clase del profesor.

Como resultado, el docente puede obtener los siguientes niveles de desempeño: i) Destacado (nota de 3,1 a 4) y que se refiere a un desempeño profesional que sobresale consistentemente con respecto a lo que se espera para el indicador evaluado; ii) Competente (nota de 2,51 a 3), referido a un desempeño profesional adecuado y que cumple con lo requerido para ejercer profesionalmente como docente. Se trata del desempeño mínimo esperado; iii) Básico (nota de 2 a 2,4), atinente a un desempeño profesional que solo ocasionalmente cumple con lo esperado en el indicador evaluado, y iv) Insatisfactorio (nota de 1 a 1,99), respecto de un desempeño con claras debilidades que afectan significativamente al papel del docente.

Aquellos docentes que obtienen un nivel de desempeño destacado o competente en la evaluación pueden postular a la Asignación Variable por Desempeño Individual (AVDI), para lo cual deben rendir una prueba de conocimientos disciplinarios y pedagógicos. Los que obtengan resultados "destacado, competente o suficiente" en la prueba AVDI, obtienen un aumento en su renta de entre un $5 \%$ y hasta un $25 \%$ sobre la renta básica mínima nacional (RBMN), lo que está en el orden de los 213 dólares mensuales (valores en que se considera la RBMN del año 2010 y el valor del dólar promedio de ese mismo año). Hasta el año 2010, casi 8.000 docentes recibieron este beneficio en Chile.

Los docentes que obtienen el resultado básico en la Evaluación Docente deben asistir a Planes de Superación Profesional, que incluyen tutorías, cursos, talleres, lecturas recomendadas y observación de clases realizadas por docentes calificados. Por último, los docentes con resultado insatisfactorio deben participar en dichos Planes y repetir su evaluación al año siguiente. Si en la segunda evaluación obtiene nuevamente el nivel de insatisfactorio, el docente debe dejar de realizar clases 
durante un año para asistir a los Planes de Superación Profesional y someterse a una tercera evaluación. Si una vez más obtiene el nivel insatisfactorio, el profesor debe dejar de pertenecer a la dotación docente.

Al año 2009 habían sido evaluados más de 50.000 profesores de escuelas municipalizadas, lo que corresponde a un $67 \%$ del total. Hasta el año 2007 , 1.050 docentes habían sido calificados alguna vez como insatisfactorios, de ellos 95 fueron calificados dos veces como insatisfactorios y 8 fueron calificados de este modo tres veces consecutivas (Araya y otros, 2010).

La parte de la evaluación que resulta central en el presente estudio es el comportamiento en el aula por parte de los profesores. Este se observa por medio de videos registrados en la clase, que son analizados por un grupo de profesionales del área de educación y — sobre la base de criterios estándar en relación con los aspectos que deben estar presentes en el aula- evaluados en los distintos ítems. Evidentemente, la observación mediante videos de una clase puede ser criticada, pues pudiera reflejar muy imperfectamente lo que es la tendencia de mediano plazo. Los profesores pueden estar nerviosos y prepararse especialmente para la clase filmada, en tanto que los alumnos también pueden ser afectados. De hecho, un $20 \%$ de los profesores evaluados en los años 2005 y 2006 señalaron que la actividad era muy difícil. Sin embargo, cerca del 80\% manifestó que el comportamiento de los alumnos era similar a lo habitual, y menos de un $10 \%$ expresó que era peor que lo habitual. Por otra parte, Lock y Strong (2010) sugieren que las evaluaciones en la sala suelen dejar de lado parte importante del desarrollo de la clase, lo que dice relación con aspectos sicológicos.

La posición adoptada en este estudio respecto de la discusión previa es que la filmación será una buena métrica del desempeño del profesor dentro de la sala de clases, en la medida en que los errores cometidos no se relacionen con otras características del educador, de los alumnos o del entorno, que afectan también al desempeño. Esto es, si las desviaciones respecto del comportamiento habitual son aleatorias, las estimaciones perderán confiabilidad, pero seguirán siendo insesgadas. En todo caso, eso se debiera reflejar en los datos y en las mismas estimaciones.

\section{III \\ Datos y resultados}

\section{Datos}

La base de datos utilizada contiene el puntaje de las distintas pruebas SIMCE de $4^{\circ}$ básico y $2^{\circ}$ medio del año 2008 y las pruebas de $8^{\circ}$ básico del año 2007 de los alumnos que asisten a escuelas municipales. Se tienen características respecto de cada alumno y de la escuela a la que asiste, entre ellas su género, la educación de los padres y el ingreso familiar. En cuanto a las características del establecimiento, se dispone de los puntajes obtenidos en la evaluación de los profesores de ese establecimiento durante los años 2005, 2006, 2007 y 2008. Además, se cuenta con los puntajes detallados que obtuvieron los profesores en los indicadores de la clase filmada.

Tales indicadores son nueve y en ellos se refleja la actuación del profesor dentro de la sala de clases y cómo son las interacciones que ocurren entre los miembros de la sala. Los indicadores son: i) focalización de los alumnos en las actividades propuestas (INDF1); ii) manejo del grupo del curso (INDF2); iii) promoción de la participación de todos los alumnos (INDF3); iv) calidad de la estructura de la clase (INDG1); v) utilización del tiempo instruccional (INDG2); vi) contribución de las actividades al logro de los objetivos (INDG3); vii) calidad de las explicaciones entregadas por el profesor (INDH1); viii) calidad de la interacción que promueve el profesor (INDH2), y ix) acompañamiento de las actividades en función del aprendizaje (INDH3).

En el gráfico 1 se aprecia la distribución de los puntajes en los distintos indicadores que tienen como rango de 0 a 4 . El área representada por las cajas muestra dónde se ubican el segundo y tercer cuartil de la población denotando poca varianza en estos, lo que en principio pudiera dificultar la pesquisa de efectos en los resultados. 
GRÁFICO 1

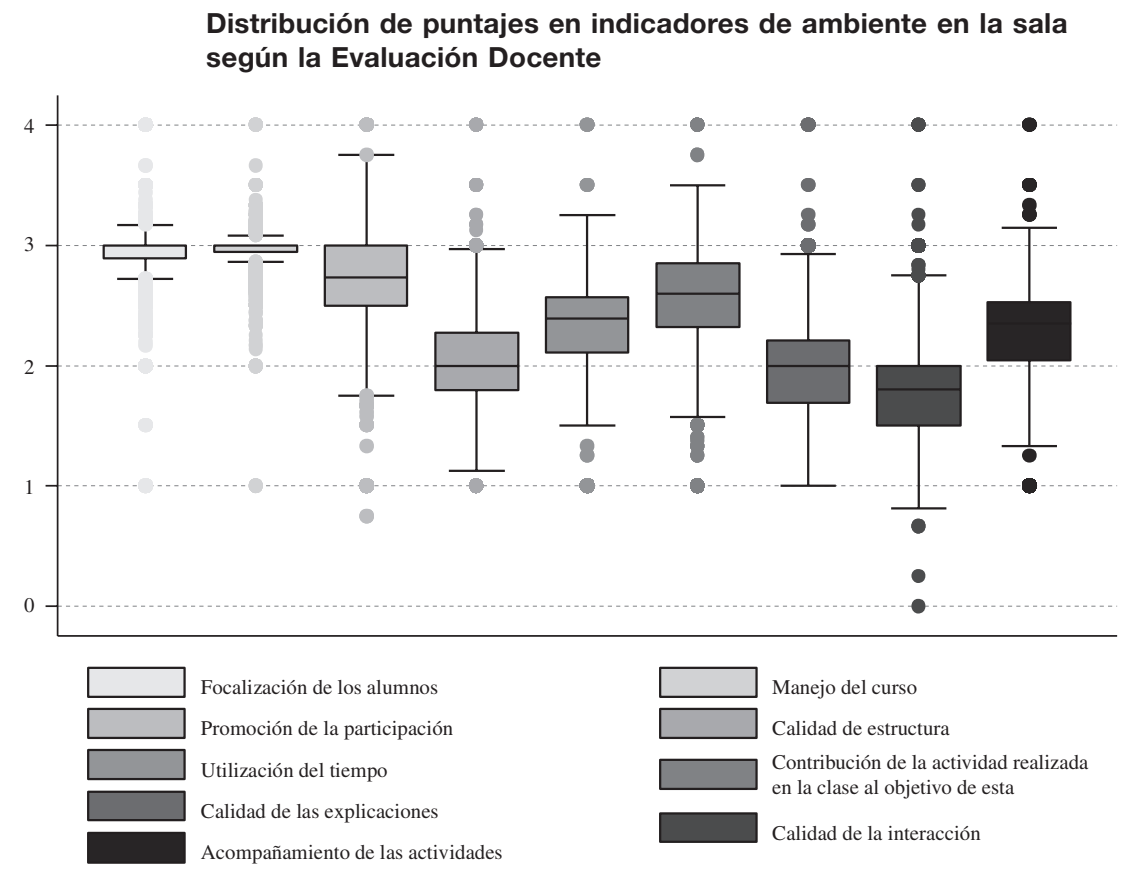

Fuente: elaboración propia sobre la base de datos de la Evaluación Docente del Ministerio de Educación (MINEDUC).

\section{El modelo de estimación}

Para estimar la influencia del ambiente de la clase en el rendimiento de los alumnos se toma en cuenta la literatura tradicional, en la que se estima que este depende de factores del entorno familiar, de la escuela (se pueden considerar aquellos relacionados con los docentes) y factores ambientales. Específicamente, en el modelo empleado en este estudio se estima como variable dependiente el desempeño del alumno, medido a través de la prueba SIMCE, y como variables explicativas, su género, su condición socioeconómica, dos medidas del efecto de los pares, la ruralidad, la calidad de los docentes - medida de acuerdo con la evaluación general del profesor (utilizando distintos instrumentos) - y, por último, el ambiente en la sala de clases, que es precisamente el factor en que se focaliza la presente investigación. De manera concreta, en el modelo por estimar se consideran las variables especificadas en (1).

$$
R_{i j}=f\left(A_{i j}, F_{i j}, P_{i j}, E_{j}, C_{j}\right)+\varepsilon_{i j}
$$

donde $R_{i j}$ es el rendimiento académico del alumno $i$ en la escuela $j ; A_{i j}$ son las características del alumno; $F_{i j}$ son las características de la familia del alumno; $P_{i j}$ son las características de los pares del alumno; $E_{j}$ son las características de la escuela $j ; C_{j}$ son las características del ambiente que hay en el establecimiento $j \mathrm{y}$, por último, $\varepsilon_{i j}$ es un error aleatorio.

Teniendo en cuenta que la teoría sobre la forma funcional más apropiada de la relación a estimar no está consensuada, y que una especificación funcional inadecuada pudiera conducir a estimaciones sesgadas o inconsistentes, se probó un conjunto de especificaciones y se compararon sobre la base del criterio de información bayesiano (BIC por sus siglas en inglés). Específicamente, se siguió el criterio desarrollado por Gideon E. Schwarz (1978), y se eligió la forma funcional que reportase el menor BIC, criterio estrechamente relacionado con la maximización de la función de verosimilitud.

Los resultados sugieren que hay gran consistencia y robustez de los distintos modelos usados, pero de acuerdo con el BIC se presenta y elabora el modelo que incluye los siguientes regresores: i) género; ii) educación de los padres; iii) ingreso familiar (lineal y cuadrático); iv) educación promedio de las madres; v) ingreso familiar promedio; vi) ruralidad; vii) puntaje promedio de los profesores de la escuela en la Evaluación Docente, y viii) nueve puntajes promedio de los indicadores de ambiente en la clase.

Existen distintos aspectos o problemas que cabe abordar en el momento de la estimación. El primero de 
ellos radica en que las observaciones no son independientes entre sí, ya que los estudiantes están agrupados en escuelas. En consecuencia, se tienen dos niveles, alumno y escuela, por lo que el término del error $\varepsilon_{i j}$ incluye un error a nivel individual del alumno, $\omega_{i j}$ y un error a nivel de establecimiento educacional, que es compartido por todos los alumnos de la escuela $\mu_{j}$.

$$
\varepsilon_{i j}=\omega_{i j}+\mu_{j}
$$

Aunque las agregaciones no reportan resultados sustancialmente diferentes, desconocer esta estructura de los datos podría conducir a estimaciones sesgadas. Si el término de error a nivel escuela, $\mu_{j}$, está correlacionado con los regresores, entonces los estimadores de mínimos cuadrados ordinarios (MCO) serán sesgados. Por otra parte, si $\mu_{j}$ es independiente de los regresores, los estimadores MCO serán insesgados, pero ineficientes.

Una posible solución a este problema es usar estimadores de efectos fijos. El inconveniente de este método para el presente propósito es que el efecto fijo es linealmente dependiente de la variable que aquí interesa, lo que haría imposible identificar el efecto.

La alternativa al uso de estimadores de efectos fijos es la estimación con efectos aleatorios. Para obtener estimadores insesgados, este método requiere de independencia entre $\mu_{j}$ y los regresores. Si esta condición se cumple, el estimador de efectos construye estimadores más eficientes (Baum, 2006).

Para probar la independencia entre $\mu_{j}$ y los regresores, se utiliza la prueba de Hausman. Si $\mu_{j}$ y los regresores están correlacionados, los estimadores de efectos fijos serán consistentes, pero los de efectos aleatorios serán inconsistentes. Por el contrario, si $\mu_{j}$ y los regresores son independientes, entonces los estimadores de efectos fijos seguirán siendo consistentes (aunque no eficientes) y los de efectos aleatorios serán consistentes y eficientes. Con la prueba de Hausman se comparan los estimadores de efectos fijos y efectos aleatorios, si estos difieren significativamente, se concluye que el supuesto de independencia es verdadero (Baum, 2006). Los resultados de la prueba de Hausman indican que la hipótesis de independencia entre $\mu_{j}$ y los regresores debe rechazarse, por lo tanto, los estimadores de efectos aleatorios, así como los de MCO, son sesgados.

Para resolver el problema se sigue a Mundlak (1978) usando el método de estimador de error cuadrático medio (Mean Square Error Estimator). La primera etapa del método consiste en estimar el rendimiento solo con aquellas variables que cambian en el interior de la escuela, como en la ecuación (3).

$$
R_{i j}=f\left(A_{i j}, F_{i j}, P_{i j}\right)+\varphi_{i j}
$$

El error estimado en la ecuación (3) incluye el error que tendría una estimación por efectos fijos, más el efecto de las variables fijas. Así, en una segunda etapa se estima una regresión entre el error estimado de (3) $\left(\widehat{\varphi_{i j}}\right)$ y las variables que no cambian en cada escuela, a través de un modelo de efectos aleatorios como en la ecuación (4).

$$
\widehat{\varphi_{i j}}=f\left(E_{j}, C_{j}\right)+\pi_{i j}
$$

Este método permite tener estimadores insesgados de los indicadores del ambiente en la sala de clases, siempre que no estén presentes otros problemas, y en particular, endogeneidad de la calidad de los profesores.

La endogeneidad de la calidad de los profesores es otro problema potencial que cabe abordar. Si los docentes que tienen mayor manejo en el aula pudieran elegir dónde educar, posiblemente preferirían las escuelas que poseen los mejores alumnos. En este caso, la causalidad iría en un sentido opuesto al que se está procurando medir y la falta de instrumentos implicaría la obtención de estimadores sesgados.

El problema de endogeneidad es uno de los más complejos de resolver, pues —en un sentido puristano tiene otra forma de solución que la aplicación de experimentos controlados o pseudoexperimentos. Se puede plantear que debido a la complejidad de los factores que interactúan, es imposible asegurar que no exista algún tipo de endogeneidad. Sin perjuicio de lo anterior, se testeó la posible endogeneidad en el modelo y se realizó una prueba de endogeneidad débil (Schaffer y Stillman, 2006). En esta prueba se estima una variable que reúne el efecto de aquellas características que influyen en el nivel docente de un establecimiento y que no han sido incluidas en el modelo (esto se obtiene como residuo de la regresión del nivel docente de los establecimientos, en función de sus otras variables que sí han sido incluidas en el modelo). Luego se evalúa la significancia que tiene esta variable estimada (las características que influyen en el nivel docente y que no han sido incluidas) al explicar el error a nivel de establecimientos que tiene el modelo original. Si esta 
variable fuese significativa, se concluiría la presencia de endogeneidad débil. Los resultados de esta prueba arrojan que la variable no es significativa, con un valor $p$ cercano a 1 , lo que sugiere que, dada la advertencia previa, no hay evidencia suficiente para sostener la hipótesis de endogeneidad.
Las estimaciones fueron realizadas respecto de las pruebas de lenguaje y matemáticas, con relación a los años 2007 y 2008 y para los cuartos y octavos básicos. En los cuadros 1 y 2 se reportan los resultados de las estimaciones según el modelo jerárquico lineal con respecto a matemáticas para cuartos y octavos básicos.

CUADRO 1

Resultados utilizando la prueba SIMCE de matemáticas de $4^{\circ}$ básico

\begin{tabular}{|c|c|c|}
\hline & $\begin{array}{c}\text { Coeficiente } \\
\text { (error estándar) }\end{array}$ & $\begin{array}{l}\text { Coeficiente } \\
\text { estandarizado }\end{array}$ \\
\hline \multicolumn{3}{|l|}{ Primer modelo } \\
\hline Mujer & $\begin{array}{l}-4,037 * * * \\
(0,303)\end{array}$ & $-0,039$ \\
\hline Educación padre & $\begin{array}{l}0,450 * * * \\
(0,042)\end{array}$ & 0,039 \\
\hline Educación madre & $\begin{array}{l}1,434 * * * \\
(0,052)\end{array}$ & 0,103 \\
\hline Ingreso (medido en $\$ 10000$ ) & $\begin{array}{l}0,232 \text { *** } \\
(0,015)\end{array}$ & 0,128 \\
\hline Ingreso al cuadrado & $\begin{array}{l}-, 001 * * * \\
(0,000)\end{array}$ & $-0,099$ \\
\hline Efecto pares educación madre & $\begin{array}{l}5,886 \text { *** } \\
(0,334)\end{array}$ & 0,186 \\
\hline Efecto pares ingreso & $\begin{array}{c}0,757 \\
(0,715)\end{array}$ & 0,012 \\
\hline Constante modelo 1 & $\begin{array}{l}136,488 * * * \\
(10,268)\end{array}$ & \\
\hline \multicolumn{3}{|l|}{$\underline{\text { Segundo modelo }}$} \\
\hline Urbano & $\begin{array}{c}-12,762 * * * \\
(0,899)\end{array}$ & $-0,098$ \\
\hline Puntaje Evaluación Docente & $\begin{array}{l}12,754 * * * \\
(1,285)\end{array}$ & 0,072 \\
\hline Focalización de los alumnos en las actividades & $\begin{array}{c}0,725 \\
(2,916)\end{array}$ & 0,002 \\
\hline Manejo del grupo curso & $\begin{array}{l}12,191 \text { *** } \\
(3,674)\end{array}$ & 0,027 \\
\hline Promoción de la participación de todos los alumnos & $\begin{array}{l}3,437 * * \\
(1,552)\end{array}$ & 0,015 \\
\hline Calidad de la estructura de la clase & $\begin{array}{l}-1,678 \\
(1,266)\end{array}$ & $-0,009$ \\
\hline Utilización del tiempo instruccional & $\begin{array}{c}1,566 \\
(1,691)\end{array}$ & 0,006 \\
\hline Contribución de las actividades a los objetivos & $\begin{array}{c}1,358 \\
(1,234)\end{array}$ & 0,006 \\
\hline Calidad de las explicaciones & $\begin{array}{c}0,976 \\
(1,721)\end{array}$ & 0,005 \\
\hline Calidad de la interacción que promueve el profesor & $\begin{array}{l}-3,187^{*} \\
(1,638)\end{array}$ & $-0,018$ \\
\hline Acompañamiento de las actividades en función del aprendizaje & $\begin{array}{c}0,246 \\
(1,950)\end{array}$ & 0,001 \\
\hline Constante modelo 2 & $\begin{array}{l}-71,811 * * * \\
(10,268)\end{array}$ & \\
\hline Número de observaciones & 104656 & \\
\hline Número de grupos & 4281 & \\
\hline $\mathrm{R}^{2}$ dentro del colegio & 0,036 & \\
\hline $\mathrm{R}^{2}$ entre colegios & 0,104 & \\
\hline $\mathrm{R}^{2}$ & 0,0787 & \\
\hline
\end{tabular}

Fuente: elaboración propia sobre la base de datos de la Evaluación Docente del Ministerio de Educación (MINEDUC).

$* \mathrm{p}<0,1 ; * * \mathrm{p}<0,05 ; * * * \mathrm{p}<0,01$. 
CUADRO 2

Resultados utilizando la prueba SIMCE de matemáticas de $2^{\circ}$ medio

\begin{tabular}{|c|c|c|}
\hline & $\begin{array}{l}\text { Coeficiente } \\
\text { (error estándar) }\end{array}$ & $\begin{array}{l}\text { Coeficiente } \\
\text { estandarizado }\end{array}$ \\
\hline \multicolumn{3}{|l|}{ Primer modelo } \\
\hline Mujer & $\begin{array}{l}-9,321 * * * \\
(0,373)\end{array}$ & $-0,08$ \\
\hline Educación padre & $\begin{array}{c}0,075^{*} \\
(0,042)\end{array}$ & 0,007 \\
\hline Educación madre & $\begin{array}{l}0,729 * * * \\
(0,059)\end{array}$ & 0,047 \\
\hline Ingreso (medido en $\$ 10000$ ) & $\begin{array}{l}0,138^{* * * *} \\
(0,017)\end{array}$ & 0,069 \\
\hline Ingreso al cuadrado & $\begin{array}{l}-0,0004 * * * \\
(0,0001)\end{array}$ & $-0,038$ \\
\hline Efecto pares educación madre & $\begin{array}{l}9,419^{* * * *} \\
(0,26)\end{array}$ & 0,287 \\
\hline Efecto pares ingreso & $\begin{array}{l}4,925^{* * * *} \\
(0,581)\end{array}$ & 0,079 \\
\hline Constante modelo 1 & $\begin{array}{l}102,367 * * * \\
(2,66)\end{array}$ & \\
\hline \multicolumn{3}{|l|}{$\underline{\text { Segundo modelo }}$} \\
\hline Urbano & $\begin{array}{l}-5,316^{*} \\
(3,14)\end{array}$ & $-0,017$ \\
\hline Puntaje Evaluación Docente & $\begin{array}{l}17,101 * * * \\
(3,736)\end{array}$ & 0,124 \\
\hline Focalización de los alumnos en las actividades & $\begin{array}{l}39,699 * * * \\
(11,98)\end{array}$ & 0,155 \\
\hline Manejo del grupo curso & $\begin{array}{l}25,453^{* *} \\
(10,79)\end{array}$ & 0,089 \\
\hline Promoción de la participación de todos los alumnos & $\begin{array}{c}3,599 \\
(4,294)\end{array}$ & 0,019 \\
\hline Calidad de la estructura de la clase & $\begin{array}{c}0,065 \\
(3,645)\end{array}$ & 0,001 \\
\hline Utilización del tiempo instruccional & $\begin{array}{l}11,485^{* * *} \\
(5,05)\end{array}$ & 0,08 \\
\hline Contribución de las actividades a los objetivos & $\begin{array}{c}-10,019 * * \\
(4,378)\end{array}$ & $-0,083$ \\
\hline Calidad de las explicaciones & $\begin{array}{c}3,79 \\
(4,398)\end{array}$ & 0,031 \\
\hline Calidad de la interacción que promueve el profesor & $\begin{array}{l}-2,752 \\
(4,182)\end{array}$ & $-0,024$ \\
\hline Acompañamiento de las actividades en función del aprendizaje & $\begin{array}{l}-0,034 \\
(6,247)\end{array}$ & $-0,0002$ \\
\hline Constante modelo 2 & $\begin{array}{c}-256,283^{* * * *} \\
(27,046)\end{array}$ & \\
\hline Número de observaciones & 74912 & \\
\hline Número de grupos & 681 & \\
\hline $\mathrm{R}^{2}$ dentro del colegio & 0,0509 & \\
\hline $\mathrm{R}^{2}$ entre colegios & 0,4905 & \\
\hline $\mathrm{R}^{2}$ & 0,2834 & \\
\hline
\end{tabular}

Fuente: elaboración propia sobre la base de datos de la Evaluación Docente del Ministerio de Educación (MINEDUC).

$* \mathrm{p}<0,1 ; * * \mathrm{p}<0,05 ; * * * \mathrm{p}<0,01$.

Los resultados de la prueba de Fisher para el conjunto de las variables que representan el clima escolar sugieren que estas pertenecen al modelo y tienen efectos relevantes. Se aprecia también que el poder predictivo del modelo es sustancialmente mayor en el caso de los cursos superiores. Así, las estimaciones muestran que para $4^{\circ}$ básico el $\mathrm{R}^{2}$ es de un $7,8 \%$, mientras que para $2^{\circ}$ medio el $\mathrm{R}^{2}$ es de un $28 \%$. Esta diferencia se debe principalmente a las disimilitudes del $\mathrm{R}^{2}$ entre escuelas: un $10 \%$ para $4^{\circ}$ básico y un $49 \%$ para $2^{\circ}$ medio.

$\mathrm{El}$ conjunto de los regresores tiene el signo y la significancia típicamente hallados en la literatura (García 
y Paredes, 2010), por lo que aparecen como controles satisfactorios. Las variables de desempeño del profesor y del ambiente en la clase son interesantes. En efecto, mientras la evaluación global del profesor es altamente relevante, de los indicadores del ambiente en la sala solo el ítem "manejo del grupo del curso" aparece como significativo en forma consistente en las regresiones de las distintas pruebas y cursos.

Este ítem se relaciona estrechamente con el propósito que el profesor quiere darle al curso, y aunque no se estima el tiempo dedicado al manejo administrativo o a la mantención del control — tiempos que se ven en la literatura como contrarios a los propósitos educativos-, parece muy probable que efectivamente el manejo del curso tenga una relación negativa con tal dedicación. La variable "manejo del grupo del curso" es consistentemente positiva y significativa en los distintos modelos. Esto parece reflejar que la habilidad del profesor para manejar el curso y alinearlo con un comportamiento deseado de este (objetivable y observable) es deseable para que se produzca el aprendizaje (Kennedy, 2005).

Con mayor precisión, la relevancia del efecto estimado del "manejo del grupo del curso" para el SIMCE de matemáticas en $4^{\circ}$ básico se aprecia en el coeficiente estandarizado de esta variable, que es de 0,27. Esto quiere decir que si se aumenta en una desviación estándar el puntaje de los profesores en el manejo del grupo $(0,35$ puntos de un total de 4), el puntaje simcE de los alumnos se acrecentaría en 0,27 desviaciones estándar (esto equivale a aproximadamente 15 puntos).

Cabe señalar también que el efecto estandarizado del manejo del curso en la prueba de matemáticas tiende a triplicarse en el caso del nivel 10 con respecto a los niveles 4 y 8 , aunque debido a la escasa varianza de las respuestas no se puede asegurar que se trata de diferencias estadísticamente significativas. En cuanto a variables como "focalización de los alumnos en las actividades", "calidad de la estructura de la clase", "contribución de las actividades a los objetivos" y "utilización del tiempo instruccional", si bien en algunos años los resultados muestran los signos y significancias esperados, ello no ocurre en forma consistente a través de las distintas pruebas, cursos y años. Por último, las variables "promoción de la participación de todos los estudiantes", "calidad de las explicaciones", "calidad de la interacción que promueve el profesor" y "acompañamiento de las actividades en función del aprendizaje" son consistentemente no significativas en lo que se refiere a explicar el desempeño de los alumnos.
Dos posibles razones pueden explicar por qué - aparte del manejo en la sala de clases - las otras variables no parecen relevantes o solo lo son para algunas pruebas. La primera es que es posible que las características señaladas efectivamente no tengan relevancia para el logro académico de los alumnos. La segunda es que el instrumento utilizado no logre captar las características relevantes, ya sea porque la observación es imperfecta o porque esos aspectos pueden ser especialmente modificados para la clase que el profesor sabe de antemano que será observada.

En procura de profundizar en la naturaleza del ambiente en la escuela, cabe preguntarse si aquello que es clave para el aprendizaje resulta manejable por el profesor o los profesores del área, o tiene que ver con un aspecto más general del ambiente de la escuela. Esto es, se puede esperar que haya escuelas que generan ambientes cálidos, en los que hay compromiso y la labor del profesor se enmarca en dicho ambiente, siendo menores sus grados de influencia.

Para probar esta hipótesis, se reemplaza la variable construida a partir del promedio de todos los profesores de la escuela, por una construida solo a partir de las observaciones a los profesores del ciclo relevante por asignatura. Así, por ejemplo, para la prueba SIMCE de matemáticas de $4^{\circ}$ básico, se consideran ahora como regresores el promedio de los puntajes obtenidos por los profesores que hacen clases de matemáticas en el primer ciclo. Evidentemente, se trata de una prueba más directa del efecto disciplinario del ambiente en la sala en que se enseña la materia, puesto que aparte de considerar a los profesores más directamente involucrados con las materias relevantes, se considera también el ciclo de estudio más cercano. En tal sentido, lo esperable es que la importancia de los coeficientes aumente significativamente.

Los resultados de estas estimaciones son sorprendentes. En general, el efecto estimado de la variable "manejo del grupo del curso" se mantiene significativo para casi todas las pruebas, pero cae la estimación puntual en casi la mitad, sin perjuicio de que el estimador no es estadísticamente menor (los intervalos de confianza para una significancia del $95 \%$ se traslapan).

Las estimaciones, al igual que en el caso anterior, se realizaron respecto de las pruebas de lenguaje y matemáticas para los años 2007 y 2008, y con relación a los cuartos básicos, octavos básicos y segundos medios. En el cuadro 3 se reportan los resultados para matemáticas en $2^{\circ}$ medio. Las estimaciones en los otros cursos y para otras pruebas siguen las mismas tendencias. 
CUADRO 3

Resultados al utilizar la prueba SIMCE de matemáticas de $2^{\circ}$ medio, considerando solo a los profesores del ramo de enseñanza media

\begin{tabular}{|c|c|c|}
\hline & $\begin{array}{l}\text { Coeficiente } \\
\text { (error estándar) }\end{array}$ & $\begin{array}{l}\text { Coeficiente } \\
\text { estandarizado }\end{array}$ \\
\hline \multicolumn{3}{|l|}{ Primer modelo } \\
\hline Mujer & $\begin{array}{l}-9,321 * * * \\
(0,389)\end{array}$ & $-0,08$ \\
\hline Educación padre & $\begin{array}{l}0,079 * \\
(0,044)\end{array}$ & 0,007 \\
\hline Educación madre & $\begin{array}{l}0,734 * * * \\
(0,061)\end{array}$ & 0,047 \\
\hline Ingreso (medido en $\$ 10000$ ) & $\begin{array}{l}0,132^{* * * *} \\
(0,017)\end{array}$ & 0,066 \\
\hline Ingreso al cuadrado & $\begin{array}{l}-0,0004 * * * \\
(0,0001)\end{array}$ & $-0,037$ \\
\hline Efecto pares educación madre & $\begin{array}{l}9,692 * * * \\
(0,269)\end{array}$ & 0,295 \\
\hline Efecto pares ingreso & $\begin{array}{l}4,692 * * * \\
(0,599)\end{array}$ & 0,075 \\
\hline Constante modelo 1 & $\begin{array}{l}99,816^{* * * *} \\
(2,772)\end{array}$ & \\
\hline \multicolumn{3}{|l|}{ Segundo modelo } \\
\hline Urbano & $\begin{array}{l}-5,562 \\
(3,709)\end{array}$ & $-0,017$ \\
\hline Puntaje Evaluación Docente & $\begin{array}{l}13,634 * * * \\
(2,291)\end{array}$ & 0,099 \\
\hline Focalización de los alumnos en las actividades & $\begin{array}{l}-2,64 \\
(4,987)\end{array}$ & $-0,01$ \\
\hline Manejo del grupo curso & $\begin{array}{l}12,523 * * \\
(5,39)\end{array}$ & 0,044 \\
\hline Promoción de la participación de todos los alumnos & $\begin{array}{c}0,861 \\
(2,862)\end{array}$ & 0,004 \\
\hline Calidad de la estructura de la clase & $\begin{array}{c}2,121 \\
(1,763)\end{array}$ & 0,021 \\
\hline Utilización del tiempo instruccional & $\begin{array}{c}0,758 \\
(2,521)\end{array}$ & 0,005 \\
\hline Contribución de las actividades a los objetivos & $\begin{array}{l}-4,506 * * \\
(1,966)\end{array}$ & $-0,037$ \\
\hline Calidad de las explicaciones & $\begin{array}{c}0,688 \\
(2,031)\end{array}$ & 0,006 \\
\hline Calidad de la interacción que promueve el profesor & $\begin{array}{l}-2,079 \\
(1,938)\end{array}$ & $-0,018$ \\
\hline Acompañamiento de las actividades en función del aprendizaje & $\begin{array}{c}2,52 \\
(2,819)\end{array}$ & 0,016 \\
\hline Constante modelo 2 & $\begin{array}{l}-67,412 * * * \\
(15,159)\end{array}$ & \\
\hline Número de observaciones & 69265 & \\
\hline Número de grupos & 602 & \\
\hline $\mathrm{R}^{2}$ dentro del colegio & 0,052 & \\
\hline $\mathrm{R}^{2}$ entre colegios & 0,4459 & \\
\hline $\mathrm{R}^{2}$ & 0,26892 & \\
\hline
\end{tabular}

Fuente: elaboración propia sobre la base de datos de la Evaluación Docente del Ministerio de Educación (MINEDUC).

$* \mathrm{p}<0,1 ; * * \mathrm{p}<0,05 ; * * * \mathrm{p}<0,01$. 
Finalmente, en el cuadro 4 se evidencia el valor de este coeficiente estandarizado para las distintas pruebas y para las dos especificaciones de los modelos estimados, es decir, usando el promedio de todos los profesores y empleando el promedio de los profesores de la asignatura y de cada ciclo.

CUADRO 4 Comparación del coeficiente indicador "Manejo grupo de curso" utilizando
el promedio del colegio comparado con el promedio del ciclo

Coeficiente estandarizado de "Manejo grupo de curso"

\begin{tabular}{|c|c|c|c|}
\hline & & Colegio & Ciclo \\
\hline $4^{\circ}$ & $\begin{array}{l}\text { Matemáticas } \\
\text { Lenguaje }\end{array}$ & $\begin{array}{l}0,027 \\
0,034 \\
\end{array}$ & $\begin{array}{l}0,023 \\
0,026 \\
\end{array}$ \\
\hline $8^{\circ}$ & $\begin{array}{l}\text { Matemáticas } \\
\text { Lenguaje }\end{array}$ & $\begin{array}{l}0,021 \\
0,032 \\
\end{array}$ & $\begin{array}{l}0,024 \\
0,022\end{array}$ \\
\hline $2^{\circ}$ medio & $\begin{array}{l}\text { Matemáticas } \\
\text { Lenguaje }\end{array}$ & $\begin{array}{l}0,089 \\
0,038\end{array}$ & $\begin{array}{l}0,044 \\
0,025\end{array}$ \\
\hline
\end{tabular}

Fuente: elaboración propia sobre la base de los datos de la Evaluación Docente entregados por el Ministerio de Educación (MINEDUC).

\section{IV}

\section{Conclusiones}

En este trabajo se ha medido y cuantificado la importancia del ambiente en las escuelas y en la sala de clases en los resultados académicos. El manejo del curso, al ser estadísticamente significativo y educacionalmente relevante, sugiere que es fundamental lograr dirigir el comportamiento del curso para alcanzar buenos aprendizajes en los alumnos. Por otra parte, se infiere de este resultado que el método utilizado para medir estas características - es decir, los videos de una clase para la Evaluación Docente- permite distinguir entre aquellos profesores que tienen la capacidad de manejar al curso y aquellos que no la tienen, y por ende, el instrumento permite a los evaluadores asignar distintas notas a aquellos docentes con distinta habilidad para manejar el curso.

Sin embargo, solo se ha considerado a una parcialidad del ambiente de la escuela y, específicamente, aquellos aspectos del ambiente escolar modificables por el profesor dentro del aula y que son perceptibles para un observador de un módulo de clases. Sin dudas que el clima de la sala de clases está mediado por muchos otros factores no observables en un video del aula, y que podrían sesgar las estimaciones en la medida en que se correlacionen con variables del modelo. En principio, no se tiene una apreciación de que efectivamente se produzca tal correlación, y por lo tanto, la interpretación de este estudio, aunque cauta, sugiere un efecto relevante del ambiente en la sala de clase, así como del desarrollo de habilidades docentes para ejercer una influencia positiva en ese ambiente.

Las implicancias de política pública sin duda tienen que ver con la formación universitaria, donde se desarrolle la personalidad y el liderazgo, así como el conocimiento de la sicología de los alumnos para saber cómo lograr manejar un grupo. Complementariamente, la disminución del tamaño de los cursos con que el profesor debe trabajar facilitaría directamente el manejo del grupo por parte del docente (Angrist y Lavy, 1999).

Finalmente, y a nivel de política desde la escuela, parece evidente que el ambiente escolar, más allá de lo que ocurre en la sala de clases, es relevante para el aprendizaje. 


\section{Bibliografía}

Angrist, J. y V. Lavy (1999), "Using Maimonides' rule to estimate the effect of class size on scholastic achievement", The Quarterly Journal of Economics, vol. 114, $\mathrm{N}^{\circ} 2$, Oxford University Press.

Arancibia, V. (1994), "La educación en Chile: Percepciones de la opinión pública y de expertos", Estudios Públicos, N 54, Santiago de Chile, Centro de Estudios Públicos.

Araya, C. y otros (2010), "Theory underlying a national teacher evaluation program", Evaluation and Program Planning, vol. 33, No 4, Amsterdam, Elsevier.

Barber, M. y M. Mourshed (2008), How the World's Best-performing School Systems Come out on Top, Londres, McKinsey and Company.

Baum, C. (2006), An Introduction to Modern Econometrics Using Stata, Stata Press.

Beyer, H. (2009), “¿Qué hacer con la educación pública?”, Estudios Públicos, N ${ }^{\circ} 114$, Santiago de Chile, Centro de Estudios Públicos.

Brunner, J.J. y C. Cox (1995), "Dinámicas de transformación en el sistema educacional de Chile", Education, Equity and Economic Competitiveness in the Americas, J.M. Puryear y J.J. Brunner (eds.), Washington, D.C., Organización de los Estados Americanos.

Cabezas, V. y otros (2013), "Los determinantes del primer trabajo para profesores de Educación Básica de la Región Metropolitana”, Evidencias para políticas públicas en educación, Sexto Concurso Fonide, Santiago de Chile.

Claro, F. (2009), "Comentarios a la presentación de Inger Enkvist en el CEP", Inger Enkvist y la educación actual, Estudios Públicos, $\mathrm{N}^{\circ} 115$, Santiago de Chile, Centro de Estudios Públicos.

Drago, J.L. y R. Paredes (2011), "La brecha de calidad en la educación chilena”, Revista CEPAL, N 104 (LC/G.2498-P), Santiago de Chile, agosto.

Edwards, D. y F. Mullis (2003), "Classroom meetings: encouraging a climate of cooperation", Professional School Counseling, vol. $7, \mathrm{~N}^{\circ} 1$.

Emmer, E. y L. Stough (2001), "Classroom management: a critical part of educational psychology, with implications for teacher education", Educational Psychologist, vol. 36, $\mathrm{N}^{\circ} 2$, Taylor $\&$ Francis.

Eyzaguirre, B. y L. Fontaine (2008), "Las escuelas que tenemos", Estudios Públicos, $\mathrm{N}^{\circ} 111$, Santiago de Chile, Centro de Estudios Públicos.

Eyzaguirre, B. y C. Le Foulon (2001), "La calidad de la educación chilena en cifras”, Estudios Públicos, № 84, Santiago de Chile, Centro de Estudios Públicos.

Fontaine, A. (2002), "Equidad y calidad de la educación: Cinco proposiciones interrelacionadas", Estudios Públicos, No 87 , Santiago de Chile, Centro de Estudios Públicos.

García, C. y R. Paredes (2010), "Reducing the educational gap: good results in vulnerable groups", Journal of Development Studies, vol. 46, $\mathrm{N}^{\circ} 3$, Taylor \& Francis.

Hausman, J. y W. Taylor (1981), "Panel data and unobservable individual effects", Econometrica, vol. 49, $\mathrm{N}^{\circ}$ 6, The Econometric Society.
Kennedy, M. (2005), Inside Teaching, How Classroom Life Undermines Reform, Harvard University Press.

León, G., J. Manzi y R. Paredes (2008), "Calidad docente y rendimiento escolar en Chile: Evaluando la evaluación", tesis, Santiago de Chile, Pontificia Universidad Católica de Chile.

Lewis, R. y otros (2008), "Students' reaction to classroom discipline in Australia, Israel, and China", Teaching and Teacher Education, vol. 24, $\mathrm{N}^{\circ} 3$, Amsterdam, Elsevier.

Lock, A. y T. Strong (2010), Social Constructionism, Cambridge, Cambridge University Press.

Malmgren, K., B. Trezek y P. Paul (2005), "Models of classroom management as applied to the secondary classroom", The Clearing House, vol. 79, $\mathrm{N}^{\circ} 1$, Taylor \& Francis.

MINEDUC (Ministerio de Educación) (2008a), Estadísticas de la educación 2008 [en línea] www.mineduc.cl.

(2008b), "Resultados nacionales 2008" [en línea] www. simce.cl.

Morris-Rothschild, B. y M. Brassard (2006), “Teachers' conflict management styles: the role of attachment styles and classroom management efficacy", Journal of School Psychology, vol. 44, $\mathrm{N}^{\circ} 2$, Amsterdam, Elsevier.

Mundlak, Y. (1978), "On the pooling of time series and cross section data", Econometrica, vol. 46, $\mathrm{N}^{\circ} 1$, The Econometric Society.

Muñoz, G. y D. Raczynski (2007), "Reforma educacional chilena: El difícil equilibrio entre la macro y la micro política", Serie Estudios Socio/Económicos, № 31, Santiago de Chile.

Muñoz, G. y J. Weinstein (2009), "Calidad para todos: La reforma educacional en el punto de quiebre" [en línea] http://www. ceppe.cl/articulos-liderazgo-y-escuelas-efectivas/174-calidadpara-todos-la-reforma-educacional-en-el-punto-de-quiebreweinstein-munoz.

Nie, F. y S. Lau (2009), "Complementary roles of care and behavioral control in classroom management: the self-determination theory perspective", Contemporary Educational Psychology, vol. 34, $\mathrm{N}^{\circ} 3$, Amsterdam, Elsevier.

OCDE (Organización para la Cooperación y el Desarrollo Económicos)/ Banco Mundial (2009), Revisión de políticas nacionales de educación, París.

Opdenakker, M.C. y J.V. Damme (2006), “Teacher characteristics and teaching styles as effectiveness enhancing factors of classroom practice", Teaching and Teacher Education, vol. 22, $\mathrm{N}^{\circ} 1$, Amsterdam, Elsevier.

Paredes, R. y J. Pinto (2009), “¿El fin de la educación pública en Chile?”, Estudios de Economía, vol. 36, N 1, Santiago de Chile, Universidad de Chile.

Ritter, J. y D. Hancock (2007), "Exploring the relationship between certification sources, experience levels and classroom management orientations of classroom teachers", Teaching and Teacher Education, vol. 23, № 7, Amsterdam, Elsevier.

Schaffer, M. y S. Stillman (2006), "XTOverid: Stata module to calculate tests of overidentifying restrictions after xtreg, xtivreg, xtivreg2, xthtaylor", Statistical Software Components, Boston College.

Schwarz, G. (1978), "Estimating the dimension of a model", The Annals of Statistics, vol. 6, $\mathrm{N}^{\circ}$ 2, Institute of Mathematical Statistics. 